\section{L'Actualité économique}

L'ACTUALITÉ ÉCONOMIQUE

L'industrie lourde en Union Soviétique (Système de direction et de planification), par SERGE MAWRISKI. Un vol., 61/4 po. x 93/4, relié, 155 pages. - LIBRAIRIE E. DROZ, 8, rue Verdaine, Genève et LIBRAIRIE MINARD, 73, rue Cardinal Lemoine, Paris $\mathrm{V}^{\mathrm{e}}$

\title{
Monique Frappier-DesRochers
}

Volume 37, numéro 3, octobre-décembre 1961

URI : https://id.erudit.org/iderudit/1001743ar

DOI : https://doi.org/10.7202/1001743ar

Aller au sommaire du numéro

Éditeur(s)

HEC Montréal

ISSN

0001-771X (imprimé)

1710-3991 (numérique)

Découvrir la revue

Citer ce compte rendu

Frappier-DesRochers, M. (1961). Compte rendu de [L'industrie lourde en Union Soviétique (Système de direction et de planification), par SERGE MAWRISKI. Un vol., 61/4 po. x 93/4, relié, 155 pages. — LIBRAIRIE E. DROZ, 8, rue Verdaine, Genève et LIBRAIRIE MINARD, 73, rue Cardinal Lemoine, Paris V $\mathrm{V}^{\mathrm{e}}$ ]. L'Actualité économique, 37(3), 562-565. https://doi.org/10.7202/1001743ar d'utilisation que vous pouvez consulter en ligne. 


\section{L'ACTUALITE ECONOMIQUE}

a perdu de son élan. Plusieurs théoriciens du monde ouvrier parmi les plus écoutés ont soustrait la nationalisation des principes sur lesquels doit s'appuyer le socialisme, et une partie importante de l'opinion publique entretient des doutes sur l'efficacité de la nationalisation comme moyen d'augmenter la production.

Cet ouvrage n'est donc pas un simple relevé des succès et des insuccès des entreprises nationalisées, mais un état consciencieux des réalisations effectuées et de leurs faiblesses, des difficultés vaincues et des problèmes encore en suspens dans la marche à la nationalisation. Il permet d'établir une comparaison avec ce qui se passe sur le continent nord-américain dans ce domaine.

Camille Martin

L'industrie lourde en Union Soviétique (Système de direc' tion et de planification), par Serge Mawriski. Un vol., 61/4 po. $\times$ 93/4, relié, 155 pages. - Librairie E. Droz, 8, rue Verdaine, Genève et Librairie Minard, 73, rue Cardinal Lemoine, Paris Ve.

Dans la collection des «Etudes d'histoire économique, politique et sociale», dirigée par MM. Jacques Freymond et Jacques L'Huillier, M. Serge Mawriski vient de publier un volume sur la planification et le système de direction de la production en U.R.S.S., notamment la production de l'industrie lourde. Nous insistons sur les sujets proposés: planification et direction de la production en général. Car l'auteur, tout en intitulant son ouvrage: L'industrie lourde en Union Soviétique, système de direction et de planification, n’en consacre pas moins la moitié (la première partie et même une section de la deuxième partie) à l'étude du sys' tème de direction et de planification de toute la production industrielle. Il est vrai que si M. Serge Mawriski avait, dès le début de son volume, étudié uniquement l'évolution de l'industrie lourde en la détachant de l'histoire du système industriel et productif en général, nous n'aurions pas saisi aussi nettement la longue évolur tion et les causes des transformations qui se sont manifestées depuis 1917.

C'est un peu l'histoire de la production à l'intérieur du système socialiste et communiste que l'auteur nous décrit dans son volume. Nous pouvons mieux accepter le titre, compte tenu de l'importance attachée à l'industrie lourde en Union Soviétique. Toutefois, nous déplorons une absence presque totale de statistiques. Cellesci auraient pu nous renseigner avec plus de précision sur la situation et l'évolution véritable de l'industrie étudiée. Cette lacune est proba. blement due au manque de renseignements disponibles à l'extérieur du rideau de fer.

En introduction, l'auteur nous explique les causes de l'intérêt porté à l'in' dustrie lourde en U.R.S.S.: 1) nécessité de l'indépendance politique et écono mique de la Russie, désireuse de passer du système capitaliste au système socialiste et communiste, ce qui a forcé les autorités à exiger des ouvriers de produire eux-mêmes leurs propres moyens de production; 2) considérations de prestige politique sur le plan mondial; 3) importance de la classe ouvrière elle-même base du parti communiste - ce qui postule une part plus grande de cette dernière au sein de la production en général; 4) théorie marxiste elle-même, qui accorde 


\section{LES LIVRES}

la priorité à l'industrie lourde, celle-ci assurant la base matérielle et technique du socialisme et son développement economique.

La première partie analyse de façon détaillée l'évolution et les transformations du système de planification et des organes de direction de toute la production en Russie et dans les Républiques fédérées. On imagine les tâtonnements qui ont affecté le système durant toute la période d'organisation. Il suffit pour cela de comparer l'économie du temps des tzars à ce qu'elle est aujourd'hui.

Les premières transformations ont été effectuées au sein des entreprises mêmes: premières nationalisations d'industries, en commençant, nous le soulignons, par les industries lourdes. Puis, essais de contrôle ouvrier de l'entreprise, gestion exclue cependant; car, l'incapacité et l'ignorance des premiers ouvriers soviétiques étaient flagrantes. Les organes de direction étaient les suivants: Conseil Supérieur de l'Économie Nationale (C.S.E.N.), organe de planification centrale industrielle; glavkhis, organes de direction par branches industrielles; Conseils de l'Économie nationale (C.E.N.) dépendant du C.S.E.N. et appliqués aux régions économiques.

Il est remarquable que les organes de planification et de direction industrielles, adoptés dès le début, n’avaient jamais perdu leurs objectifs initiaux, en dépit de transformations quant à leur forme même.

Nous ne mentionnerons pas toutes les transformations rapportées par l'auteur. Nous nous bornerons à suivre la ligne de pensée du système, à souligner les erreurs et les réussites, et à en faire la critique. Après la période de guerre et de reconstruction, période qui a ralenti considérablement la production en général et fait «dévier les plans, on essaye de relever et d'encourager l'économie par un retour au capitalisme dirigé. Un plan directeur apparaît: le Gosplan qui, lui aussi, à travers des transformations successives, est demeuré jusqu'à aujourd'hui l'instrument de planification par excellence. On va même jusqu'à laisser l'offre et la demande fluctuer librement sur le marché. Cependant, l'industrie lourde demeure contrôlée et planifíé. À l'époque 1921.25 et aux époques suivantes (soit jusqu'en 1957), les organes centraux de direction se multiplient tellement que leurs fonctions respectives ne sont pas bien délimitées et empiètent les unes sur les autres. Il existe trop de subordination des organes inférieurs aux organes - supérieurs, ce qui nuit à l'efficacité. L'auteur, sans mentionner de chiffres cependant, nous fait remarquer que le niveau de production, en 1925, en était au même point qu'en 1913. On tente de stimuler la production par le nouveau principe du Khoraschet qui, dans nos systèmes libéraux, demeure le principe de base de toute organisation de l'entreprise: une entreprise doit fonctionner avec profit, sinon elle est vouée à l'échec!

L'époque des plans quinquennaux débute en 1926, plans qui seront, de façon passagère, septennaux. Comme nous le soulignions, les organes de direction se métamorphosent et sont corrigés à chaque nouvelle réunion du Parti - ou presque! - L'époque de libéralisme «dirigé, ne dure pas longtemps; au contraire, la tendance est très vite à un resserrement du contrôle. Le désir de décen. traliser subsiste, mais il est mis en sommeil. La crainte du retour à la propriété 


\section{L'ACTUALITE ECONOMIQUE}

privée, et aussi probablement le manque de connaissance et la pénurie d'hommes compétents dans les organes subalternes, empêchent le parti communiste d'accorder plus de souplesse et de liberté aux organes de planification et de direction de la production. Tout reste rigoureusement contrôlé par le parti central. Les essais de décentralisation ne prendront vraiment corps qu'en 1957, à la suite d'une réforme nécessaire à l'essor de la production.

En 1957, des changements notoires et définitifs sont effectués. La multiplication des organismes centraux de direction empêchait, jusqu'alors, une bonne organisation de l'industrie et même des entreprises: la direction se trouvait trop éloignée de la production, et les chefs d'entreprises recevaient souvent des ordres contradictoires, en raison de la complexité des autorités d'où ils émanaient. Un principe du début est à nouveau remis à l'honneur: le principe de la direction de la production par régions économiques («principe territorial»). On décentralise ainsi la direction opérationnelle, tout en renforçant les directives planificatrices et les pouvoirs de la direction générale. L'industrie lourde elle-même est décentralisée et cela pour la première fois depuis l'avènement du socialisme.

Les organes centraux de direction (Conseil des Ministres de l'U.R.S.S. et des Républiques) reçoivent l'aide d'organismes techniques dont la tâche se limite aux problèmes économiques (Gosplan secondé de divers comités). Puis le «principe territorial» conduit à une division du pays en régions économiques et administratives: chaque région doit s'efforcer de vivre de ses propres ressources, en accordant toutefois la priorité à une production spécialisée quelconque. Dans chaque région, les Conseils de l'Économie nationale (C.E.N.) ou sounarkhozes, assurent la direction de toutes les branches productrices. Ils sont aidés à leur tour par des comités techniques et économiques. Au bas de l'échelle administrative viennent les entreprises dont les directeurs sont nommés par les sounarkhozes et représentent le gouvernement.

$\mathrm{C} e$ nouveau système d'administration et de planification de la production met en application les principes théoriques de Lénine et de Marx, soit:

1) Le centralisme démocratique, système en vertu duquel la direction générale reste centralisée, tout en permettant une décentralisation opérationnelle (Lénine).

2) Le principe de direction territoriale de l'économie. Marx a toujours souligné l'importance du développement économique par régions.

3) L'unité de direction. Ce principe est difficile à réaliser, compte tenu des interventions du parti dans le domaine économique et le domaine syndical.

4) Le khoraschet, dont nous avons parlé plus haut.

Trois chapitres décrivent en détails le travail du Gosplan et l'élaboration des plans trimestriels et quinquennaux. À notre époque de planification et de capitalisme dirigé, l'expérience russe vaut la peine qu'on s'y arrête. La méthode des bilans passés et prévisionnels, même impossible à appliquer avec autant de rigueur dans nos économies capitalistes, demeure une source de renseignements féconds.

En conclusion, l'auteur revient aux problèmes de l'industrie lourde, et il se demande quel a été le rôle et l'importance de cette industrie dans le passé. Au cours 


\section{LES LIVRES}

du présent volume, la priorité de l'industrie lourde dans le développement industriel et agricole de I'U.R.S.S. s'est trouvée soumise à plusieurs reprises. À la fin de son étude, M. Mawriski remonte à Marx pour trouver l'explication d'une telle priorité. En effet, le philosophe allemand a recommandé le développement toujours prioritaire des moyens de production, base de tout développement éco nomique en système socialiste et communiste. Cependant, l'écart toujours plus grand entre la production agricole et industrielle d'une part, et la production de l'industrie légère et de l'industrie lourde d'autre part, a fait douter certains éco. nomistes russes de la véracité actuelle d'un tel principe. En effet, maintenant que l'ère du socialisme se trouve atteinte, et que l'on approche lentement du plein épanouissement du communisme, ces économistes ont demandé au parti s'il n'était pas plus utile de chercher à mettre un peu plus l'accent sur les biens de consommation, pour chercher à combler l'écart. Mais le Conseil des Ministres, s'appuyant sur la théorie marxiste, s'y est formellement opposé.

L'auteur explique les raisons de cette priorité considérée nécessaire au régime socialiste: recherche de la puissance politique et militaire, respect de la thérie marxiste, importance de l'industrie lourde soviétique dans les relations de la Russie avec les autres pays communistes et les pays sous-développés. Puis, il analyse les trois possibilités à venir du développement de la production industrielle et agricole en U.R.S.S.: a) priorité accordée à l'industrie lourde sur l'industrie légère et agricole; b) développement parallèle de l'industrie lourde et de l'industrie légère et agricole; c) développement prioritaire, du moins pour un certain temps, des industries secondaires de consommation sur l'industrie lourde.

Il conclut en signalant les défauts de la théorie économique soviétique, en ce qui concerne le développement futur de l'industrie lourde. Il la juge imprécise - la philosophie communiste est hégélienne, il ne faut pas Youblier - tour à tour trop concrète ou trop abstraite. Les économistes ont limité leurs recherches à la phase d'évolution dans laquelle ils se trouvaient, nous dit M. Mawriski. Il n'existe pas de fondement théorique de nature économique, pour la phase actuelle, considérée comme celle du passage du socialisme au communisme. Les citations des économistes, à propos d'une priorité quelconque dans le dévelop. pement économique des différentes industries, se révèlent contradictoires. Le futur apparaît donc extrêmement incertain.

Monique Frappier-DesRochers

\section{De Thomas More à Chaptal, par Marguerite Leblanc. Un} vol., $61 / 2$ po. $\times 91 / 2$, broché, 169 pages. - Editrons Cujas, 19, rue Cujas, Paris, 1961.

Il est particulièrement difficile de rendre compte du contenu de l'ouvrage de Marguerite Leblanc - et l'analyste le déplore - . L'idée d'où est partie Marguerite Leblanc doit être retenue plus que le contenu du livre. Les salles de statistiques et d'économie politique de la Faculté de Droit et des Sciences Éco. nomiques de Paris ont eu la chance insigne de recevoir, en dotation, la majeure 